\title{
Effects of 24-week add-on treatment with ciclesonide and montelukast on small airways inflammation in asthma.
}

\section{$\operatorname{AUTHOR}(\mathrm{S})$ :}

Nakaji, Hitoshi; Petrova, Guergana; Matsumoto, Hisako; Iwata, Toshiyuki; Ito, Isao; Oguma, Tsuyoshi; Inoue, Hideki; ... Kanemitsu, Yoshihiro; Niimi, Akio; Mishima, Michiaki

\section{CITATION:}

Nakaji, Hitoshi ... [et al]. Effects of 24-week add-on treatment with ciclesonide and montelukast on small airways inflammation in asthma.. Annals of allergy, asthma \& immunology 2013, 110(3): 198-203.e3

\section{ISSUE DATE:}

2013-03

URL:

http://hdl.handle.net/2433/173625

\section{RIGHT:}

(C) 2013 American College of Allergy, Asthma \& Immunology. Published by Elsevier Inc.; この論文は出版社版でありません。引用の際には出版 社版をご確認ご利用ください。; This is not the published version. Please cite only the published version. 
1 Effects of 24-week add-on treatment with ciclesonide and montelukast on small airways

2 inflammation in asthma

3

4 *Hitoshi Nakaji ${ }^{1,2}$, ${ }^{*}$ Guergana Petrova ${ }^{1}$, Hisako Matsumoto ${ }^{1}$, Toshiyuki Iwata ${ }^{1}$, Isao Ito ${ }^{1}$,

5 Tsuyoshi Oguma ${ }^{1}$, Hideki Inoue ${ }^{1}$, Tomoko Tajiri ${ }^{1}$, Tadao Nagasaki ${ }^{1}$, Yoshihiro Kanemitsu ${ }^{1}$,

6 Akio Niimi $^{1,3}$, Michiaki Mishima ${ }^{1}$

7

$8{ }^{1}$ Department of Respiratory Medicine, Graduate School of Medicine, Kyoto University, Kyoto,

9 Japan

$10{ }^{2}$ Department of Respiratory Medicine, Wakayama Red Cross Hospital, Wakayama, Japan

$11{ }^{3}$ Division of Respiratory Medicine, Department of Medical Oncology and Immunology, Nagoya

12 City University School of Medical Sciences, Nagoya, Japan

$13 *$ HN and GP equally contributed to this study

14 Trial registration; Registry ID UMIN000001083

15

16 Corresponding author: Hisako Matsumoto, $\mathrm{MD}, \mathrm{PhD}$

17 Department of Respiratory Medicine

18 Postgraduate School of Medicine, Kyoto University

1954 Kawahara-cho, Shogoin, Sakyo-ku, Kyoto 606-8507, Japan

20 Telephone: +81-75-751-3830; Fax: +81-75-751-4643

21 E-mail: hmatsumo@kuhp.kyoto-u.ac.jp

22

23 


\section{Authors' contributions}

$25 \mathrm{HN}$ recruited and managed the patients, collected, analyzed and interpreted the data, and

26 prepared the manuscript. GP collected, analyzed and interpreted the data, and wrote the draft.

27 HM conceived the study, recruited and managed the patients, collected, analyzed and interpreted

28 the data, and revised the manuscript. TI collected and analyzed data and prepared the part of the

29 draft. AN and II recruited the patients, collected the data, and contributed to the edition of the

30 manuscript. TO and HI performed IOS measurements and collected the data and prepared the

31 part of the draft. TT, TN, and YK measured exhaled nitric oxide and analyzed and interpreted the

32 data, and prepared the part of the draft. MM contributed to the discussion of the data and critical

33 revision of the manuscript. 


\section{Abstract}

36 Background: Eosinophilic inflammation of the small airways is a key process in asthma that

37 often smolders in treated patients. The long-term effects of add-on therapy on the persistent

38 inflammation in the small airways remain unknown.

39 Objective: To examine the effects of add-on therapy with either ciclesonide, an inhaled

40 corticosteroid with extrafine particles, or montelukast on small airway inflammation.

41 Methods: Sixty patients with stable asthma receiving inhaled corticosteroid treatment were

42 enrolled in a randomized, open-label, parallel comparison study of 24-week add-on treatment

43 with ciclesonide or montelukast. Patients were randomly assigned to 3 groups: ciclesonide $(\mathrm{n}=$

44 19), montelukast $(\mathrm{n}=22)$ and no add-on as controls $(\mathrm{n}=19)$. At baseline and at weeks 4,12 and

45 24, extended nitric oxide analysis; pulmonary function tests, including impulse oscillometry; blood eosinophil counts; and asthma control tests (ACTs) were performed.

47 Results: A total of 18 patients in the ciclesonide group, 19 in the montelukast group and 15 in 48 the control group completed the study and were analysed. With repeated-measures analysis of

49 variance, ciclesonide produced a significant decrease in alveolar nitric oxide and a significant

50 improvement in ACT scores over time. Montelukast produced significant decreases in alveolar

51 nitric oxide concentrations and blood eosinophil counts over time and slightly improved ACT

52 scores, whereas no such changes were observed in the control group. Alveolar nitric oxide

53 concentrations with ciclesonide and reactance area at low frequencies with montelukast produced

54 greater improvements over time compared with control.

55 Conclusions: Ciclesonide add-on therapy and montelukast add-on therapy may act differently,

56 but both separately can improve small airway abnormalities and provide better asthma control. 
58 Funding; funded by none

59

60 Key words: add-on treatment, alveolar nitric oxide, asthma control, ciclesonide, montelukast,

61 small airways

62

63 
64

65

66

67

68

69

70

71

72

73

74

75

76

77

78

79

80

81

82

83

84

85

86

\section{Introduction}

Asthma is a chronic inflammatory disease of the airways characterized by variable, recurring symptoms and reversible airflow obstruction. The immunohistopathologic features include infiltration of eosinophils and lymphocytes, mast cell activation and epithelial cell injury. To date, pathological ${ }^{1,2}$, physiologic ${ }^{3}$ and radiologic findings ${ }^{4}$ have provided sufficient evidence to support not only large but also small airways involvement in inflammation and airflow obstruction, particularly in patients with severe asthma ${ }^{5,6}$.

Recently, it was found that eosinophilic inflammation of the small airways could be assessed by determining alveolar nitric oxide concentrations ${ }^{7,8}$. Small airway inflammation as assessed by alveolar nitric oxide concentrations is increased in patients with refractory asthma ${ }^{8}$ and those with nocturnal asthma ${ }^{9}$ and is associated with disease severity ${ }^{10,11}$ and small airways dysfunction ${ }^{11}$. Of note, $20 \%$ of asthmatic patients have increased alveolar nitric oxide concentrations despite treatments with inhaled corticosteroids (ICSs) and long-acting $\beta_{2}$ agonists ${ }^{12}$. Alveolar nitric oxide concentrations can also predict a future risk of disease exacerbation ${ }^{13}$. These findings suggest that, even in apparently stable patients taking ICSs, additional treatment targeting the small airways may lead to reaching total asthma control.

Few studies have evaluated the changes in alveolar nitric oxide concentrations based on either an uncorrected ${ }^{7}$ or corrected ${ }^{14}$ model of add-on medication for persistent inflammation of the small airways. Previous studies found that oral prednisolone ${ }^{10}$, but not double doses of ICSs ${ }^{8}$ could decrease alveolar nitric oxide concentrations. These results suggest that alveolar nitric oxide concentrations may be resistant to a simple ICS dose elevation. In steroid-naive patients, however, extrafine particle hydrofluoroalkane-ciclesonide resulted in decreased alveolar nitric oxide concentrations ${ }^{15}$ and hydrofluoroalkane-beclomethasone propionate 
87 improved peripheral airway dysfunction ${ }^{16}$. Collectively, an extrafine particle ICS is expected to

88 decrease alveolar nitric oxide concentrations when they are used as an add-on medication.

89 Leukotriene receptor antagonists (LTRAs) that are administered systemically are another

90 medication that are supposed to decrease alveolar nitric oxide concentrations. Treatment with

91 montelukast for 4 weeks improved small airway obstruction in steroid-naive patients, which

92 resulted in a decrease in regional air trapping ${ }^{17}$. So far published study data of an add-on LTRA

93 to ICS therapy for 3 to 8 weeks with regard to alveolar nitric oxide concentrations have been

94 conflicting ${ }^{18,19}$. These effects require confirmation with a longer-term study.

95 For this study, we hypothesized that prolonged add-on therapy to the ICS treatment with

96 either ciclesonide or montelukast would have beneficial effects on the persistent inflammation of

97 the small airways and would improve pulmonary function. To test this hypothesis, our primary

98 objectives were to examine the effects of this add-on therapy on alveolar nitric oxide

99 concentrations and to compare its effects on small airways in patients with stable asthma who

100 had not been previously treated with extrafine particle ICSs or LTRAs. 


\section{Methods}

102 The full details of the study methods are given in the eMethods. In brief, adult patients with

103 stable asthma who regularly visited our outpatient asthma clinic were enrolled from April 2008

104 to August 2011. Asthma was diagnosed according to American Thoracic Society criteria ${ }^{20}$.

105 Patients were included if they were classified as being in treatment steps 2 to 5 of ICS treatment

106 according to the Global Initiative for Asthma guidelines ${ }^{21}$. These patients had no exacerbations

1073 months before enrollment, had alveolar nitric oxide concentrations of $5.0 \mathrm{ppb}$ or higher, and

108 were either never-smokers or ex-smokers who had smoked fewer than 5 pack-years and had

109 stopped more than 1 year before. The threshold level for uncorrected alveolar nitric oxide

110 concentrations was set at $5.0 \mathrm{ppb}$; this value was the average minus $1 \mathrm{SD}$ of uncorrected alveolar

111 nitric oxide concentrations of 70 patients with asthma taking ICSs in our previous study ${ }^{22}$.

Exclusion criteria were current or previous use of extrafine particle ICSs or LTRAs.

113 Patients were also excluded if, during the study period, any adverse effects of the add-on therapy

114 or asthma exacerbations, including mild exacerbations, defined as an increased need for rescue

115 use of short-acting $\beta_{2}$-agonists, were noted.

This study was approved by the ethics committees of our institute and was registered in

117 UMIN Clinical Trials Registry (Registry Identified UMIN000001083). Written informed consent 118 was obtained from all participants.

Design and Measurements

This was a randomized, open-label, parallel comparison study of 24-week add-on

122 treatment with either inhaled ciclesonide or montelukast. Patients were randomly assigned to 3

123 treatment groups: inhaled ciclesonide, $400 \mu \mathrm{g}$ once daily add-on (ciclesonide group); 
124 montelukast, $10 \mathrm{mg}$ once daily add-on (montelukast group); and control group, who were taking

125 current medication only. At weeks 0 (baseline), 4, 12, and 24 (end of study period) the patients

126 underwent extended nitric oxide analysis and pulmonary function tests, including tests with an

127 impulse oscillometry system (IOS), spirometry, and a nitrogen single-breath wash out test. At the

128 same time points, patients completed an asthma control test (ACT) questionnaire comprising 5

129 questions with a best possible score of $25^{23}$ and were given a rhinitis symptom score (RSS), a

130 self-assessment questionnaire comprising 4 questions, the responses to which were ranked on a

131 Likert-type scale with a maximum of 5 points per answer. The RSS was determined based on the

132 Japanese Guideline for Allergic Rhinitis (best score, 20) ${ }^{24}$ (eTable 1).

At the start and end of the study period, blood samples were obtained for blood

134 eosinophil counts and serum high sensitivity C-reactive protein ${ }^{25}$, serum eosinophil cationic

135 protein, ${ }^{26}$ and serum YKL-40, a chitinase like protein ${ }^{27}$. Blood samples for eosinophil cationic

136 protein determinations were collected in SST tubes (Becton Dickinson, Mountain View,

137 California) and were processed as previously described ${ }^{26}$. YKL-40 levels were determined using

138 an enzyme-linked immunosorbent assay kit (Quidel, San Diego, California) following the

139 manufacturer's instructions 27.

Nitric oxide levels were determined with a chemiluminescence analyzer (NOA 280;

141 Sievers, Boulder, Colorado) according to current guidelines, and as previously described alveolar

142 nitric oxide concentrations are provided as noncorrected ${ }^{7}$ and corrected values using a trumpet-

143 shaped model with axial back diffusion (eMethods) ${ }^{14}$.

$144 \quad$ After nitric oxide measurements, patients underwent prebronchodilator and

145 postbronchodilator (ie, inhalation of $200 \mu \mathrm{g}$ of salbutamol) pulmonary function tests. 
147 Respiratory Society ${ }^{28}$. A nitrogen single-breath washout test was performed only before the

148 inhalation of salbutamol to assess ventilation inhomogeneity by measuring the slope of phase 3

149 of the nitrogen washout curve $\left(\Delta \mathrm{N}_{2}\right)$.

150 Respiratory impedance was determined by IOS using a Jaeger MasterScreen, $\operatorname{IOS}^{\mathrm{TM}}$

151 (Erich Jaeger, Hoechberg Germany) that met standard recommendations (eMethods). 16, 22

\section{Statistical analysis}

154 For sample size determinations, we originally sought to enroll 90 patients based on previous 155 findings ${ }^{15,17,19}$. However, as described in the "Results" section, we decided to stop patient 156 enrollment at 60 because of the more frequent occurrence of exacerbations in the control group, 157 although these were mild.

158 Statistical analysis used JMP 6.00 (SAS Institute Inc., Cary, North Carolina) on a per159 protocol basis. For non-normally distributed results, comparisons were made by the Kruskal160 Wallis test, Fisher exact test or Wilcoxon signed-rank test as appropriate. For normally 161 distributed results, comparisons were made by analysis of variance (ANOVA) and the paired $t$ 162 test. Two-way repeated-measures ANOVA was used to assess the variations among the 3 163 treatment modalities and at different time points. For cases with unequal variations in the 164 treatment modalities, only 1-way repeated-measures ANOVA within 1 treatment group was used. 165 For correlation analysis, the Spearman rank-correlation test was used. Data are expressed as 166 mean $\pm \mathrm{SD} . \mathrm{P} \leq 0.05$ were considered statistically significant. 
168 Results

169

170

171

172

173

174

175

176

177

178

179

180

181

182

183

184

185

186

187

188

189

190

\section{Enrollment, Dropout, and Exacerbation Rates and Baseline Characteristics}

Sixty asthmatic patients were enrolled in this study and randomly assigned to the groups: 19 in the ciclesonide group, 22 in the montelukast group, and 19 in the control group (Fig 1).The reasons for patient dropout were as follows: in the ciclesonide group, 1 patient had a possible adverse effect (urticaria); in the montelukast group, 3 patients had possible adverse effects ( 2 experienced mild gastrointestinal discomfort and they preferred to discontinue use of the medication and 1 patient had mildly elevated transaminase levels); and in the control group, 3 had mild asthma exacerbations and they preferred to intensify medications and 1 patient discontinued ICS treatment following a general practitioner's advice. As a result 18 patients in the ciclesonide group, 19 in the montelukast group, and 15 in the control group completed the study and were analyzed thereafter (Table 1). For these patients, adherence to the add-on and current medications was satisfactory, which was confirmed by 2 of the authors (H.N. and H.M.) on each visit by checking the residual number of medications.

When the exacerbation frequencies were compared between the 19 patients in the control group and the 41 patients in the add-on therapy groups and assuming that the 5 patients who dropped out for reasons other than exacerbation would complete the protocol without exacerbation, the control group had a significantly higher rate of exacerbation $(\mathrm{p}=0.03$; by Fisher exact test). The baseline patient characteristics, ICS doses, and biomarkers, including fractional exhaled nitric oxide (FeNO) and alveolar nitric oxide concentrations, were not significantly different among the 3 patients who later experienced mild exacerbations and the other 57 patients. 


\section{ACT scores and RSSs}

192 By 1-way ANOVA, there was a significant improvement in ACT scores during the treatment 193 period within the ciclesonide group ( $\mathrm{p}=0.02$; Fig 2$)$, and there was a trend for improvement 194 within the montelukast group $(\mathrm{p}=0.08)$. When subscores for the ACT components were separately analyzed in the ciclesonide group, subscores for ACT question 3 concerning nocturnal

196 symptoms and question 5 for self-rating were marginally and insignificantly improved over time $197(\mathrm{p}=0.05$ and $\mathrm{p}=0.06$, respectively). Because of the unequal variations among the 3 treatment 198 modalities, we did not conduct 2-way ANOVA for the ACT scores. Details on ACT scores 199 across the treatment steps are presented in eTable 2.

Among the 3 groups, neither the proportions of patients with allergic rhinitis nor their

201 baseline RSSs differed. However, a significant difference was seen in the time trends for RSS

202 among the 3 treatment modalities ( $\mathrm{p}=0.004$; eFig 1); in particular, using 2-way ANOVA, 203 significant differences were seen for the symptom of nasal obstruction $(\mathrm{p}=0.046)$. When comparing 2 different treatment modalities in a post hoc analysis, the montelukast group exhibited a significantly better time trend for the RSS than the control group $(\mathrm{p}<0.001)$ and a

206 trend for better scores than the ciclesonide group $(\mathrm{p}=0.07$; eFig 1$)$. A significant increase in 207 RSS over time was found only in the montelukast group ( $\mathrm{p}<0.001$, by 1 -way ANOVA). 210 nitric oxide concentrations in either treatment group. 
213 No significant differences were found in the time trends for FeNO at an expiratory flow rate of

$21450 \mathrm{~mL} / \mathrm{s}$ among the 3 treatment modalities or within each of the groups (results not shown).

The time trends for uncorrected alveolar nitric oxide concentrations were significantly

216 different among the 3 treatment groups $(\mathrm{p}=0.048$, by 2 -way ANOVA). When comparing 2

217 different treatment modalities in a post hoc analysis, the ciclesonide group had a greater decrease

218 in alveolar nitric oxide concentrations over time than the control group $(\mathrm{p}=0.03$, by 2 -way

219 ANOVA). By 1-way ANOVA, alveolar nitric oxide concentrations in the control group did not

220 change during the study period, whereas in both of the add-on treatment groups, alveolar nitric

221 oxide concentrations significantly decreased over time ( $\mathrm{p}=0.01$ for the ciclesonide and

222 montelukast groups; Fig 3).

223 For corrected alveolar nitric oxide concentrations, 1-way ANOVA showed that there was 224 an insignificant decrease over time in the ciclesonide group $(\mathrm{p}=0.06)$.

225

226

\section{Pulmonary Function Tests}

227 None of the spirometry indices, $\Delta \mathrm{N}_{2}$, or IOS indices of respiratory resistance at $5 \mathrm{~Hz}\left(\operatorname{Rrs}_{5}\right)$,

228 respiratory resistance at $20 \mathrm{~Hz}\left(\mathrm{Rrs}_{20}\right)$, or respiratory reactance at $5 \mathrm{~Hz}\left(\mathrm{Xrs}_{5}\right)$ revealed any

229 difference among the 3 treatment modalities during the treatment period regardless of

230 prebronchodilator or postbronchodilator conditions. No significant changes were observed within 231 any of the 3 groups (data not shown).

A significant difference was found in the time trends for the reactance area (AX) among

233 the 3 treatment modalities ( $p=0.04$, by 2 -way ANOVA). The AX levels in the montelukast

234 group improved over time when compared with the control group ( $\mathrm{p}=0.05$, by 2-way ANOVA;

235 Fig 4). For Rrs5-Rrs 20 , 2-way ANOVA was not used because of the unequal variations among 
236 the 3 treatment modalities; however, 1-way ANOVA revealed that there was a trend for a change

237 over time in the ciclesonide group $(\mathrm{p}=0.09)$.

Although there were associations between corrected alveolar nitric oxide concentrations

239 and IOS indices of $\mathrm{AX}$ or $\mathrm{Rrs}_{5}-\mathrm{Rrs}_{20}$ at baseline $(\mathrm{r}=0.30, \mathrm{p}<0.05$ for both, $\mathrm{n}=52)$, no

240 associations were found between changes in pulmonary function data from baseline to the end of

241 the treatment period and changes in alveolar nitric oxide concentrations or corrected alveolar

242 nitric oxide concentrations in either treatment group.

243

$244 \quad$ Blood Test Results

245 Blood samples were obtained at baseline and at the end of the treatment period to determine

246 blood eosinophil counts and serum levels of eosinophil cationic protein, high sensitivity C-

247 reactive protein, and YKL-40. No significant changes were found in these tests results between

248 the beginning and the end of the treatment period, except for the montelukast group in which the

249 eosinophil counts significantly declined after treatment $(2.9 \pm 2.2 \%$ at 24 weeks $)(\mathrm{p}=0.02$, paired

$250 \quad t$ test).

251 


\section{Discussion}

253 To the best of our knowledge, this is the first long-term study that clarified the benefits and

254 potential role of add-on therapy with either ciclesonide of extrafine particle ICS or montelukast

255 in steroid-treated patients with stable asthma. Ciclesonide may have attenuated smoldering

256 inflammation of the small airways and significantly improved asthma control over time.

257 Montelukast ameliorated the remnant dysfunction of the small airways and reduced nasal

258 symptoms and blood eosinophil counts. To a lesser extent than ciclesonide, montelukast also

259 improved smoldering inflammation of the small airways.

Alveolar nitric oxide concentration is an established marker of small airway

261 inflammation and is correlated with eosinophil counts in bronchoalveolar lavage fluid ${ }^{8}$. In the

262 ciclesonide group, alveolar nitric oxide concentrations significantly decreased over time when

263 compared with the control group and the ciclesonide intragroup analysis. Our data confirmed

264 earlier findings of the effects of 5-week treatment with ciclesonide on alveolar nitric oxide

265 concentrations in steroid-naïve patients ${ }^{15}$ and reinforced the advantage of extrafine particle ICSs

266 to treat smoldering inflammation of the small airways, even in patients already taking ICSs.

267 There remains the possibility that the addition of ciclesonide to the patients' current medication

268 may have exerted anti-inflammatory effects via the increase in the total amount of ICS, which

269 may have suppressed the remnant inflammation throughout the airways. However, this is

270 unlikely because FeNO at $50 \mathrm{~mL} / \mathrm{s}$ did not change over time. Taken the results of the previous

271 short-term study and current study together, ciclesonide would be capable of treating the small

272 airways potentially because of its particles size, which was sufficiently small to reach the

273 peripheral airways. 
In contrast to uncorrected alveolar nitric oxide concentrations, corrected alveolar nitric

275 oxide concentrations only showed a trend toward being decreased in the ciclesonide group $(\mathrm{p}=$

276 0.06, 1-way ANOVA). Although corrected alveolar nitric oxide concentrations reflect airway

277 dysfunction 22,29 , as do alveolar nitric oxide concentrations, corrected alveolar nitric oxide

278 concentrations do not reflect disease severity ${ }^{14,22}$ or asthma control status ${ }^{29}$. It is also not

279 increased during asthma exacerbations in adults ${ }^{30}$, a finding that is in contrast to several lines of

280 evidence for alveolar nitric oxide concentrations. Although alveolar nitric oxide concentrations

281 are contaminated with bronchial nitric oxide, potentially from small conducting airways where

282 diffusion begins to replace bulk flow, our findings on alveolar nitric oxide concentrations imply

283 that relatively small airways, albeit not actual peripheral airways, are still important in the

284 management of asthma.

Studies of add-on medication using LTRAs that have evaluated changes in alveolar nitric

286 oxide concentrations in persistent inflammation of the small airways reported inconsistent

287 findings. Previous add-on studies of montelukast to fluticasone ${ }^{18}$ or fluticasone and salmeterol

288 treatment ${ }^{12}$ did not find any significant benefits for montelukast with regard to decreases in

289 alveolar nitric oxide concentrations after montelukast add-on therapy. However, these earlier

290 studies were relatively short-term, with treatment periods of only 3 to 4 weeks. Yasui et al.

291 investigated pranlukast use in patients with stable asthma and found significant decreases in both

292 corrected and uncorrected alveolar nitric oxide concentrations after 8-week crossover of add-on

293 therapy with pranlukast ${ }^{19}$. In agreement with that study, we found that alveolar nitric oxide

294 concentrations in the montelukast group decreased during the 24-week add-on period, although

295 these levels were not significantly different from the control group. As with the ciclesonide

296 group, FeNO at $50 \mathrm{~mL} / \mathrm{s}$ did not change over time. These findings indicate that add-on treatment 
297 with LTRAs for longer than 8 weeks suppresses the remnant inflammation in the small airways.

298 In addition, our intervention study that covered the 2 seasons for allergic rhinitis (spring and

299 autumn) provided additional evidence of the established benefit of montelukast on allergic

300 rhinitis ${ }^{31}$ and justified a role for LTRA in the therapy for patients with stable asthma with

301 concomitant allergic rhinitis, even those with minimal symptoms.

Symptoms and airway obstruction are integral to the definition of asthma, and represent important components for assessing asthma control in both clinical practice and clinical trials.

304 Therefore, one of the end points in our study was ACT scores. Despite the disadvantage in 305 adherence to inhalation use, as reported previously on adherence to LTRA of $67.7 \%$ vs. $33.8 \%$ 306 for ICS ${ }^{32}$, ACT scores significantly improved over time in the ciclesonide group. In addition, 307 there was a marginal improvement in the subscore of ACT question 3 concerning nocturnal 308 symptoms in the ciclesonide group. To date, a number of studies have confirmed that 309 eosinophilic inflammation worsens in patients with nocturnal asthma, particularly in the 310 peripheral airways ${ }^{33}$. Lehtimaki et $\mathrm{al}^{9}$ reported that nocturnal symptoms in asthmatic patients

311 were related to higher alveolar nitric oxide concentrations. These results are in accordance with 312 our results showing that ciclesonide add-on treatment reduced inflammation in the small airways, 313 as assessed by alveolar nitric oxide concentrations, and improved nocturnal symptoms, as

314 assessed by ACT subscores. Care must be taken when interpreting these findings, however, 315 because the minimally important difference in ACT scores that reflects a clinically meaningful 316 change is considered to be 3 points ${ }^{34}$, and the increase in ACT composite scores in our 317 ciclesonide group did not achieve this. Despite this minimal change, these statistically significant 318 changes would still favour add-on therapy for patients with seemingly stable asthma. 
We did not find any significant changes in spirometry function results or $\Delta \mathrm{N}_{2}$ between

320 the control and therapy add-on groups, which may seem unexpected. Spirometry is not sensitive

321 enough to detect early small airway involvement because the small airways are pathways of very

322 low resistance and only contribute to approximately $10 \%$ of the total airway resistance ${ }^{35}$. Instead

323 of using $\Delta \mathrm{N}_{2}$, ventilation heterogeneity within conductive and acinar airways could have been

324 separately assessed using a nitrogen multiple-washout test ${ }^{36}$. Another possible reason could be

325 that our patients had already good pulmonary function, so that changes in alveolar nitric oxide

326 concentrations were not reflected in the airway function. However, in the montelukast group, the

$327 \mathrm{AX}^{16,22}$ significantly decreased over time when compared with the control group, as was found

328 in our previous intervention study in steroid-naïve patients ${ }^{16}$. Montelukast may have reversed

329 remodeling in the airway walls by reducing airway smooth muscle layer thickening and

330 subepithelial fibrosis in long-term treatment, as has been shown in an animal model ${ }^{37}$. More

331 significant findings might be expected in extended studies in a larger number of patients.

A limitation of our study was that it was a parallel, open-label, and unblinded study,

which might have influenced subjective measures, such as asthma symptoms and rescue use of

334 short-acting $\beta_{2}$ agonists. Another issue is the use of 2 different inhalers for corticosteroids,

335 although we achieved good adherence in the ciclesonide group. In future studies with more

336 patients and longer treatment periods, this issue could be resolved.

In addition, we may have missed some patients with occult inflammation in the small

338 airways by excluding those with alveolar nitric oxide concentrations less than $5 \mathrm{ppb}$, given that

339 some patients who have high FeNO and low alveolar nitric oxide concentrations exhibit

340 paradoxical increases in alveolar nitric oxide concentrations after treatment ${ }^{38}$, possibly because

341 of dilatation of constricted small airways from terminal to respiratory bronchioles. However, by 
342 setting this threshold for alveolar nitric oxide concentrations during patient enrollment, the

343 changes of alveolar nitric oxide concentrations in this study could be simply interpreted.

Finally, from the ethical standpoint, we stopped enrollment at 60 patients because of a

345 higher, albeit mild, exacerbation rate in the control group, which was consistent with the finding

346 that elevated alveolar nitric oxide concentration was associated with risk of asthma

347 exacerbation ${ }^{13}$. Thus, some of the insignificant findings, particularly of the pulmonary function

348 data in this study, may be due to lesser statistical power. Lack of associations between the

349 changes in alveolar nitric oxide concentrations and changes in pulmonary function data or ACT

350 scores might be another issue. However, we did not set the sample size to seek significant

351 associations between changes in alveolar nitric oxide concentrations and any other clinical

352 indices because of their potentially large variations during the treatment period, although

353 alveolar nitric oxide concentrations, pulmonary function, and ACT were intuitively thought to

354 behave in parallel. Despite these limitations, the current findings of a decrease in alveolar nitric

355 oxide concentrations with add-on treatment are sufficient to be used as a future reference when

356 intensifying treatment with extrafine particle ICS or LTRA add-on therapy, even in patients with

357 seemingly stable asthma who are receiving ICS treatment but still have evidence of small

358 airways inflammation as assessed by alveolar nitric oxide concentrations.

359 We conclude that ciclesonide and montelukast may act differently but that both

360 separately can improve small airway abnormalities (eTable 3). By coadministration of these

361 medications, cumulative effects on inflammation and small airways function can be expected and

362 should be clarified in a future study. We can achieve additional benefits by treating inflammation

363 of the small airways in patients with stable asthma to reach the ultimate asthma treatment goal:

364 ideal control. 


\section{Acknowledgments}

367 The authors are grateful to Ms Aya Inazumi and Ms Yuko Maeda for their technical assistance.

368 
369

370

371

372

373

374

375

376

377

378

379

380

381

382

383

384

385

386

387

388

389

390

391

392

393

394

395

396

397

398

399

\section{References}

1. Hamid Q, Song Y, Kotsimbos TC, et al. Inflammation of small airways in asthma. J Allergy Clin Immunol. 1997;100: 44-51.

2. Carroll N, Cooke C, James A. The distribution of eosinophils and lymphocytes in the large and small airways of asthmatics. Eur Respir J. 1997;10: 292-300.

3. in 't Veen JC, Beekman AJ, Bel EH, Sterk PJ. Recurrent exacerbations in severe asthma are associated with enhanced airway closure during stable episodes. Am J Respir Crit Care Med. 2000;161: 1902-1906.

4. Ueda T, Niimi A, Matsumoto H, et al. Role of small airways in asthma: investigation using high-resolution computed tomography. J Allergy Clin Immunol. 2006;118: 10191025.

5. Johnson JR, Hamid Q. Appraising the small airways in asthma. Curr Opin Pulm Med. 2012;18: 23-28.

6. Contoli M, Kraft M, Hamid Q, et al. Do small airway abnormalities characterize asthma phenotypes? In search of proof. Clin Exp Allergy. 2012;42: 1150-1160.

7. Tsoukias NM, George SC. A two-compartment model of pulmonary nitric oxide exchange dynamics. J Appl Physiol. 1998;85: 653-666.

8. Berry M, Hargadon B, Morgan A, et al. Alveolar nitric oxide in adults with asthma: evidence of distal lung inflammation in refractory asthma. Eur Respir J. 2005;25: 986991.

9. Lehtimaki L, Kankaanranta H, Saarelainen S, Turjanmaa V, Moilanen E. Increased alveolar nitric oxide concentration in asthmatic patients with nocturnal symptoms. Eur Respir J. 2002;20: 841-845.

10. Gelb AF, Taylor CF, Nussbaum E, et al. Alveolar and airway sites of nitric oxide inflammation in treated asthma. Am J Respir Crit Care Med. 2004;170: 737-741.

11. van Veen IH, Sterk PJ, Schot R, et al. Alveolar nitric oxide versus measures of peripheral airway dysfunction in severe asthma. Eur Respir J. 2006;27: 951-956.

12. Gelb AF, Taylor CF, Shinar CM, Gutierrez CA, Zamel N. Effect of fluticasone 250 microg/salmeterol 50 microg and montelukast on exhaled nitric oxide in asthmatic patients. Can Respir J. 2008;15: 193-198. 
400

401

402

403

404

405

406

407

408

409

410

411

412

413

414

415

416

417

418

419

420

421

422

423

424

425

426

427

428

429

13. Gelb AF, Flynn Taylor C, Shinar CM, Gutierrez C, Zamel N. Role of spirometry and exhaled nitric oxide to predict exacerbations in treated asthmatics. Chest. 2006;129: 1492-1499.

14. Condorelli P, Shin HW, Aledia AS, Silkoff PE, George SC. A simple technique to characterize proximal and peripheral nitric oxide exchange using constant flow exhalations and an axial diffusion model. J Appl Physiol. 2007;102: 417-425.

15. Cohen J, Douma WR, ten Hacken NH, et al. Ciclesonide improves measures of small airway involvement in asthma. Eur Respir J. 2008;31: 1213-1220.

16. Yamaguchi M, Niimi A, Ueda T, et al. Effect of inhaled corticosteroids on small airways in asthma: investigation using impulse oscillometry. Pulm Pharmacol Ther. 2009;22: 326-332.

17. Zeidler MR, Kleerup EC, Goldin JG, et al. Montelukast improves regional air-trapping due to small airways obstruction in asthma. Eur Respir J. 2006;27: 307-315.

18. Fritscher LG, Rodrigues MT, Zamel N, Chapman KR. The effect of montelukast on exhaled nitric oxide of alveolar and bronchial origin in inhaled corticosteroid-treated asthma. Respir Med. 2009;103: 296-300.

19. Yasui H, Fujisawa T, Inui N, et al. Impact of add-on pranlukast in stable asthma; the additive effect on peripheral airway inflammation. Respir Med. 2012;106: 508-514.

20. Standards for the diagnosis and care of patients with chronic obstructive pulmonary disease (COPD) and asthma. This official statement of the American Thoracic Society was adopted by the ATS Board of Directors, November 1986. Am Rev Respir Dis. 1987;136: 225-244.

21. Global Initiative for Asthma Management and Prevention: National Institutes of Health, National Heart, Lung and Blood Institute, 2006.

22. Matsumoto H, Niimi A, Jinnai M, et al. Association of alveolar nitric oxide levels with pulmonary function and its reversibility in stable asthma. Respiration. 2011;81: 311-317.

23. Nathan RA, Sorkness CA, Kosinski M, et al. Development of the asthma control test: a survey for assessing asthma control. J Allergy Clin Immunol. 2004;113: 59-65.

24. Okubo K, Kurono Y, Fujieda S, et al. Japanese guideline for allergic rhinitis. Allergol Int. 2011;60: 171-189. 
430 25. Takemura M, Matsumoto H, Niimi A, et al. High sensitivity C-reactive protein in asthma. 431 Eur Respir J. 2006;27: 908-912.

432 26. Matsumoto H, Niimi A, Minakuchi M, Izumi T. Serum eosinophil cationic protein levels measured during exacerbation of asthma: characteristics of patients with low titres. Clin Exp Allergy. 2001;31: 637-643.

27. Otsuka K, Matsumoto H, Niimi A, et al. Sputum YKL-40 Levels and Pathophysiology of Asthma and Chronic Obstructive Pulmonary Disease. Respiration. 2012;83: 507-519.

28. ATS/ERS recommendations for standardized procedures for the online and offline measurement of exhaled lower respiratory nitric oxide and nasal nitric oxide, 2005. Am J Respir Crit Care Med. 2005;171: 912-930.

29. Mahut B, Trinquart L, Le Bourgeois M, et al. Multicentre trial evaluating alveolar NO fraction as a marker of asthma control and severity. Allergy. 2010;65: 636-644.

30. Gelb AF, George SC, Silkoff PE, et al. Central and peripheral airway/alveolar sites of

31. Lagos JA, Marshall GD. Montelukast in the management of allergic rhinitis. Ther Clin Risk Manag. 2007;3: 327-332.

33. Kraft M, Djukanovic R, Wilson S, Holgate ST, Martin RJ. Alveolar tissue inflammation in asthma. Am J Respir Crit Care Med. 1996;154: 1505-1510.

34. Schatz M, Kosinski M, Yarlas AS, et al. The minimally important difference of the Asthma Control Test. J Allergy Clin Immunol. 2009;124: 719-723 e711.

452 35. Macklem PT. The physiology of small airways. Am J Respir Crit Care Med. 1998;157: S181-183.

454 36. Verbanck S, Schuermans D, Vincken W. Inflammation and airway function in the lung 455 periphery of patients with stable asthma. J Allergy Clin Immunol. 2010;125: 611-616.

38. Van Muylem A, Kerckx Y, Michils A. Acinar effect of inhaled steroids evidenced by exhaled nitric oxide. J Allergy Clin Immunol. 2010;126: 730-735 e732. 


\section{$463 \quad$ Figure legends}

464 Figure 1. Registration and randomization

465 Figure 2. Asthma control test (ACT) scores in the 3 study groups. * Significant changes in ACT

466 scores within the ciclesonide add-on group ( $\mathrm{p}=0.02$, by 1 -way analysis of variance).

467 Figure 3. Alveolar nitric oxide concentrations in the 3 study groups. *Significant difference in 468 the time trends for alveolar nitric oxide concentrations among the 3 treatment modalities $(\mathrm{p}=$ 4690.048 , by 2 -way analysis of variance [ANOVA] $). \dagger$ Significant changes in alveolar nitric oxide 470 concentrations in the ciclesonide add-on group ( $\mathrm{p}=0.03$ vs the control group, by 2 -way

471 ANOVA) ( $\mathrm{p}=0.01$, by 1 -way ANOVA). $\$$ Significant changes within montelukast add-on group $472 \quad(\mathrm{p}=0.01$, by 1-way ANOVA $)$.

473 Figure 4. Reactance area (AX) levels in the 3 study groups. *Significant difference in the time 474 trends for $\mathrm{AX}$ levels among the 3 treatment modalities $(\mathrm{p}=0.04$, by 2 -way analysis of variance 475 [ANOVA] $)$, posthoc analysis between the montelukast add-on and control groups $(\mathrm{p}=0.05$, by 476 2-way ANOVA).

477 
Figure 1
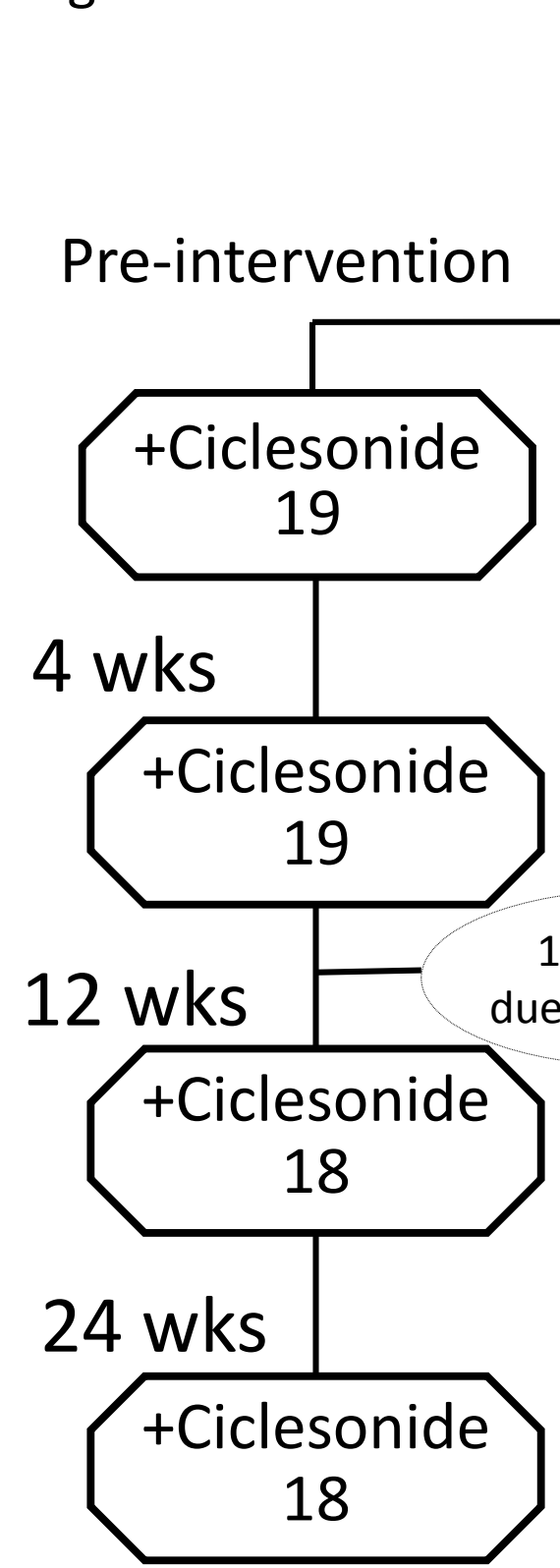

1 drop out due to urticaria

\section{Entry}

Randomization

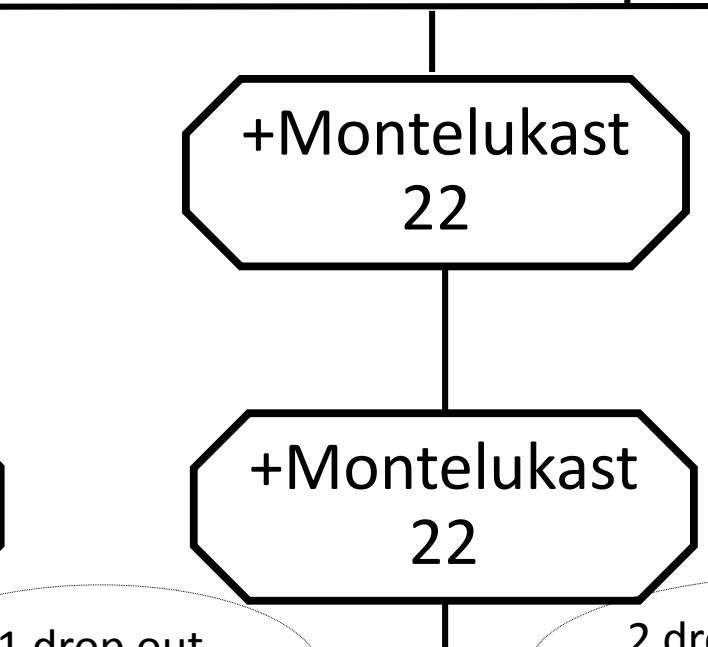

\section{drop out due to mild} gastro-intestinal discomfort

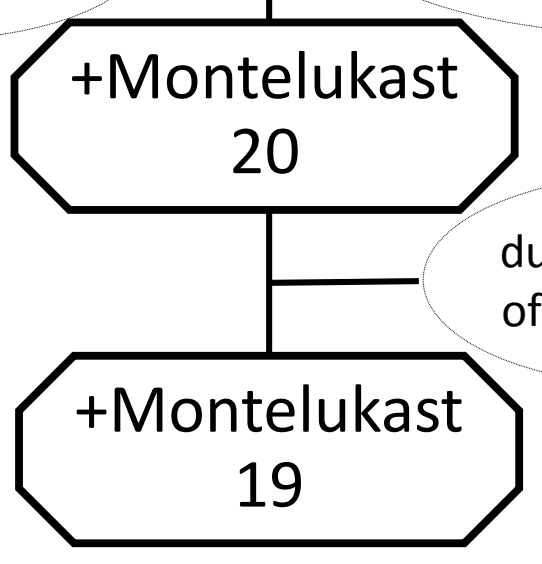

1 drop out due to elevation of transaminase

19

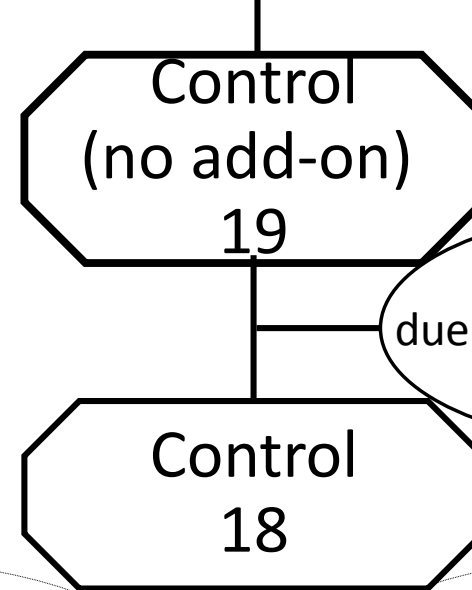

1 drop out due to protocol violation

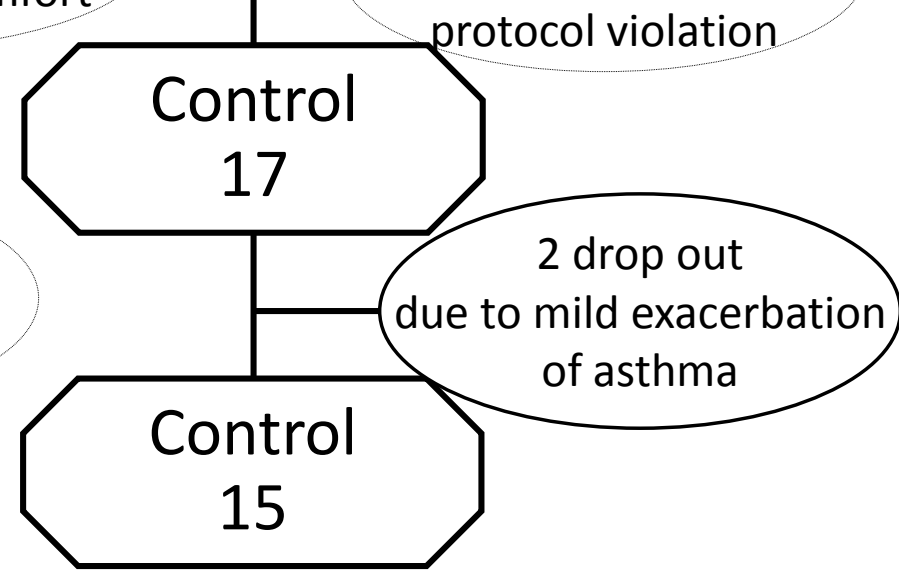




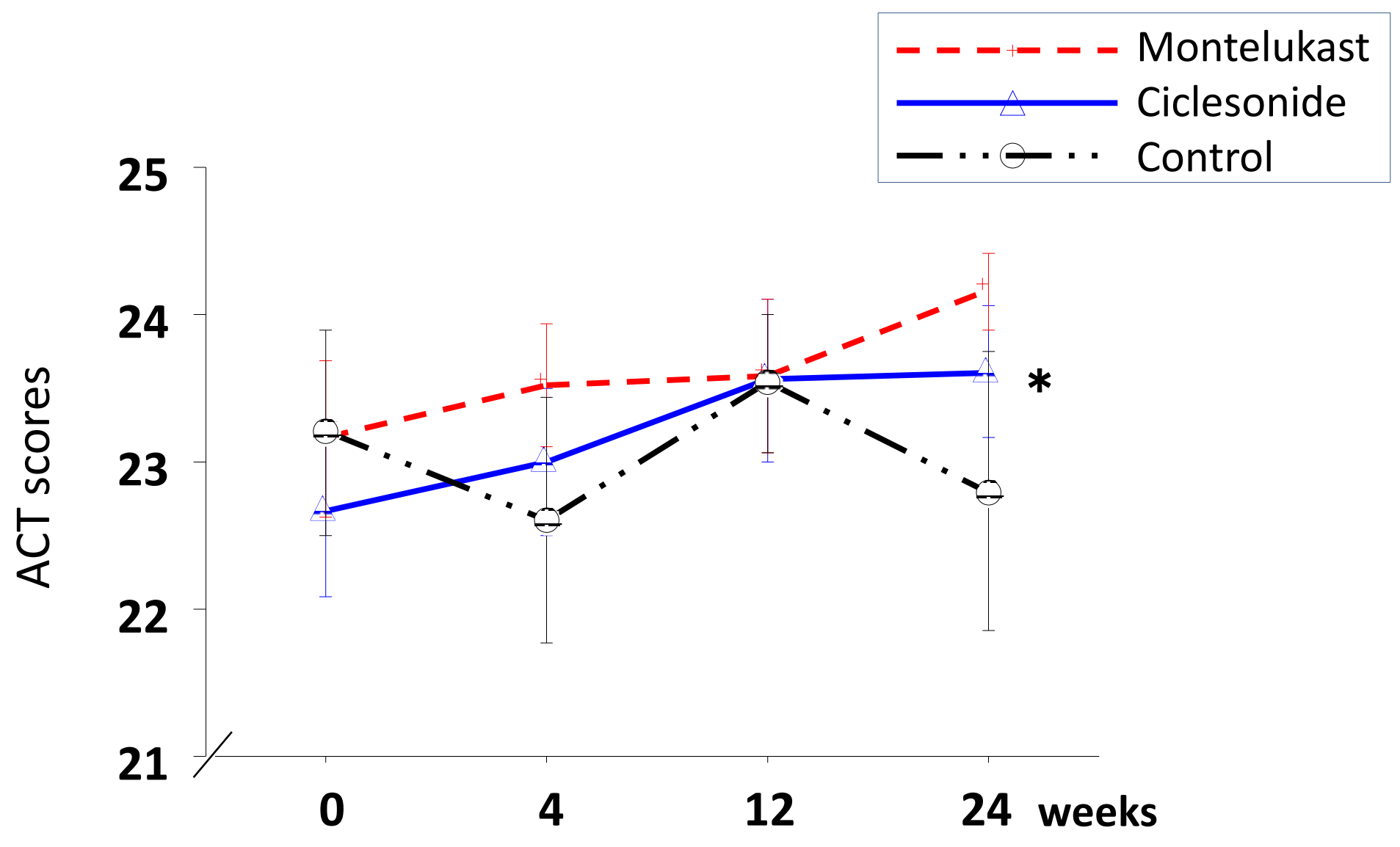

Figure 2 


\section{- - - - - - Montelukast \\ Ciclesonide \\ $-\cdots \in \cdots$ Control}

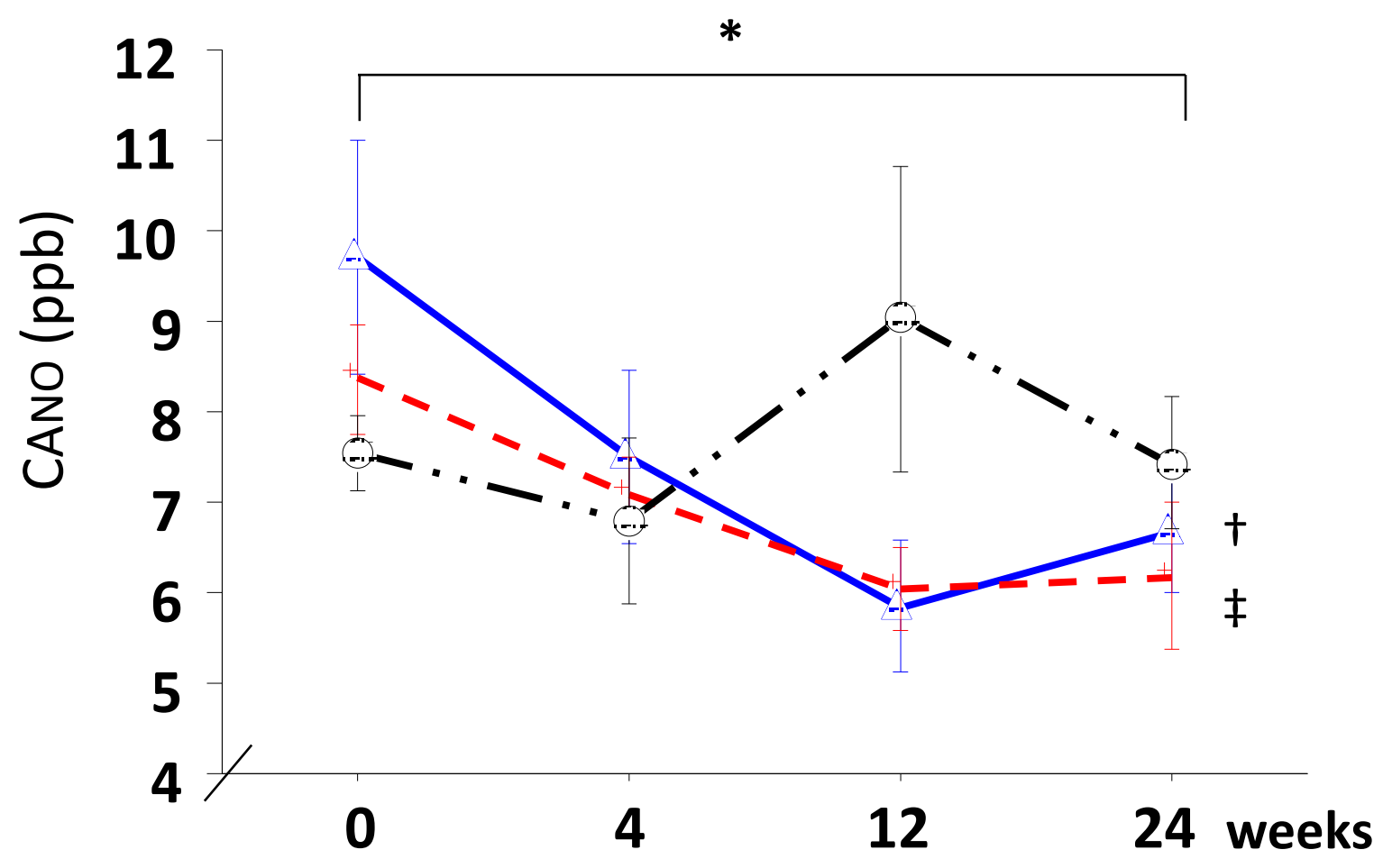

Figure 3 


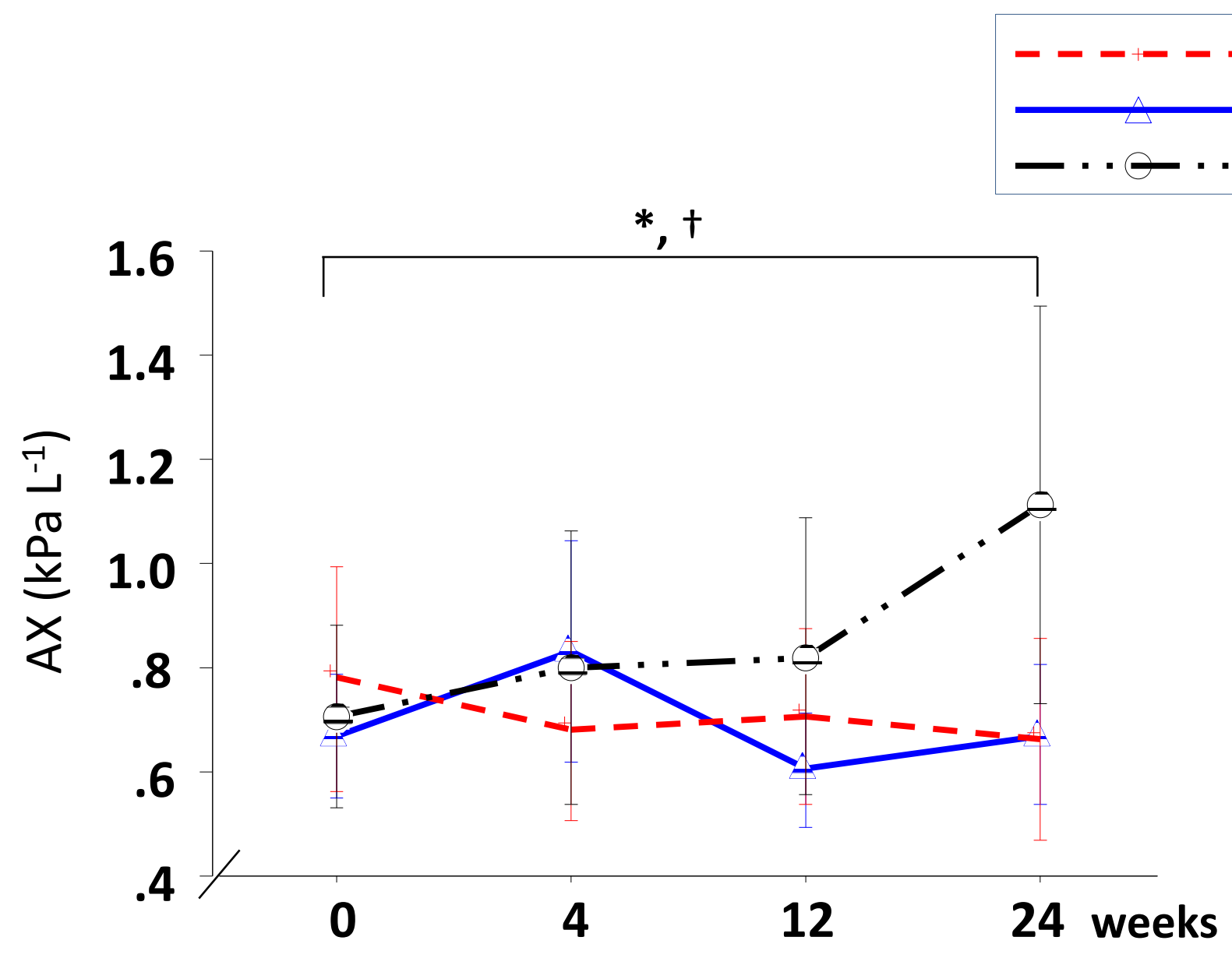

Figure 4 
Table 1. Characteristics of the study patients

481

482

483

484

485

486

487

488

489

490

491

492

493

494

495

496

497

498

499

500

501

502

503

504

505

506

507

508

509

510

511

512

513

514

515

516

517

Ciclesonide group Montelukast group Control group

( $\mathrm{n}=18$

$(\mathrm{n}=19) \quad(\mathrm{n}=15)$

Female / male

$13 / 5$

$64.5 \pm 9.9$

$13 / 6$

$9 / 6$

Age, y

$6 / 11 / 1 / 0$

$61.8 \pm 10.6$

$57.4 \pm 21.1$

Treatment Step 2/ 3/ 4/ $5^{1)}$

Smoking history

(never / ex-smoker)

$17 / 1$

$6 / 10 / 3 / 0$

$9 / 3 / 2 / 1$

Atopy (yes / no) ${ }^{2)}$

$10 / 8$

$15 / 4$

$12 / 3$

$12 / 7$

$7 / 8$

Total IgE, IU/mL

$120(7-25000)$

159 (8-1900)

$86(8-760)$

$361 \pm 263$

$353 \pm 174$

$333 \pm 222$

$11 / 7$

$10 / 9$

$6 / 9$

$3 / 16$

$1 / 14$

$44.5 \pm 36.4$

$37.5 \pm 15.7$

$42.4 \pm 32.1$

$9.7 \pm 5.6$

$8.4 \pm 2.7$

$7.5 \pm 1.6$

Corrected alveolar nitric oxide

$$
\text { concentrations, ppb }
$$

$7.0 \pm 5.5$

$93.9 \pm 17.5$

$5.7 \pm 3.3$

$5.0 \pm 2.0$

$\mathrm{FEV}_{1}, \%$ predicted

$74.7 \pm 18.6$

$93.7 \pm 20.3$

$94.7 \pm 23.8$

$\mathrm{FEV}_{1} / \mathrm{FVC}, \%$

$1.8 \pm 1.7$

$74.2 \pm 18.2$

$73.6 \pm 24.6$

$\Delta \mathrm{N}_{2}, \%$

$0.43 \pm 0.15$

$1.8 \pm 1.7$

$2.1 \pm 2.2$

$\operatorname{Rrs}_{5}, \mathrm{kPa} \mathrm{sL}^{-1}$

$0.35 \pm 0.11$

$0.40 \pm 0.13$

$0.40 \pm 0.14$

$\operatorname{Rrs}_{20}, \mathrm{kPa} \mathrm{sL}^{-1}$

$0.08 \pm 0.05$

$0.31 \pm 0.09$

$0.33 \pm 0.10$

$\mathrm{Rrs}_{5}-\mathrm{Rrs}_{20}, \mathrm{kPa} \mathrm{sL}^{-1}$

$-0.14 \pm 0.06$

$0.09 \pm 0.07$

$0.07 \pm 0.07$

$\mathrm{Xrs}_{5}, \mathrm{kPa} \mathrm{sL}^{-1}$

$\mathrm{AX}, \mathrm{kPa} \mathrm{L}^{-1}$

$0.67 \pm 0.51$

$-0.14 \pm 0.06$

$-0.14 \pm 0.07$

ACT score

$22.7 \pm 2.5$

$0.78 \pm 0.91$

$0.71 \pm 0.68$

Rhinitis symptom score

$16.9 \pm 2.1$

$23.2 \pm 2.3$

$23.2 \pm 2.7$

Blood eosinophils, \%

$5.3 \pm 3.9$

$16.4 \pm 2.1$

$17.2 \pm 1.7$

Serum ECP, $\mu \mathrm{g} / \mathrm{L}$

$16.6 \pm 17.6$

$4.7 \pm 2.9$

$4.0 \pm 2.5$

Serum hsCRP, mg/dL

$0.21 \pm 0.37$

$11.4 \pm 11.1$

$15.0 \pm 15.5$

Serum YKL-40, ng/dL

$115.2 \pm 86.0$

$0.10 \pm 0.20$

$0.14 \pm 0.17$

Data are presented as number or mean $\pm \mathrm{SD}$, except for IgE, which is presented as median (range); $>>0.05$ for all characteristics according to the analysis of variance, the Kruskal-Wallis test or Fisher's exact test. 1) According to the 2006 Global Initiative for Asthma guidelines, 2) Atopy was determined based on the presence of specific serum IgE antibodies to at least 1 common inhalant allergen, including cat dander, dog dander, weed pollens, grass pollens, molds, or house dust mite, 3 ) equivalent to fluticasone proprionate 


\section{E-Supplement material}

519

520 Effects of 24-week add-on treatment with ciclesonide and montelukast on small airways

521 inflammation in asthma

522

523 *Hitoshi Nakaji ${ }^{1,2}$, * Guergana Petrova ${ }^{1}$, Hisako Matsumoto ${ }^{1}$, Toshiyuki Iwata ${ }^{1}$, Isao Ito ${ }^{1}$, 524 Tsuyoshi Oguma ${ }^{1}$, Hideki Inoue ${ }^{1}$, Tomoko Tajiri ${ }^{1}$, Tadao Nagasaki ${ }^{1}$, Yoshihiro Kanemitsu ${ }^{1}$, 525 Akio Niimi ${ }^{1,3}$, Michiaki Mishima ${ }^{1}$

526

$527{ }^{1}$ Department of Respiratory Medicine, Graduate School of Medicine, Kyoto University, Kyoto, 528 Japan

$529{ }^{2}$ Department of Respiratory Medicine, Wakayama Red Cross Hospital, Wakayama, Japan

$530{ }^{3}$ Division of Respiratory Medicine, Department of Medical Oncology and Immunology, Nagoya

531 City University School of Medical Sciences, Nagoya, Japan

$532 * \mathrm{HN}$ and GP equally contributed to this study

533 Trial registration; Registry ID UMIN000001083

534

535 Corresponding author: Hisako Matsumoto, $\mathrm{MD}, \mathrm{PhD}$

536 Department of Respiratory Medicine

537 Postgraduate School of Medicine, Kyoto University

53854 Kawahara-cho, Shogoin, Sakyo-ku, Kyoto 606-8507, Japan

539 Telephone: +81-75-751-3830; Fax: +81-75-751-4643

540 E-mail: hmatsumo@kuhp.kyoto-u.ac.jp 
eMethods

Adult patients with stable asthma who regularly visited our outpatient asthma clinic were enrolled from April 2008 to August 2011. Asthma was diagnosed according to American Thoracic Society criteria based on a history of recurrent episodes of wheezing and chest tightness, with or without cough, and documented airway reversibility with a bronchodilator or hyperresponsiveness to inhaled methacholine ${ }^{\mathrm{e}}$.

Nitric oxide levels were determined with a chemiluminescence analyzer (NOA 280; Sievers, Boulder, Colorado) according to current guidelines and as previously described ${ }^{\mathrm{e} 2}$. The analyzer was daily calibrated with gas without nitric oxide and a standard concentration of 640 ppb nitric oxide. Lower detection limit for nitric oxide was $2 \mathrm{ppb}$. The concentrations were determined using a data analysis program (NOA Analysis ${ }^{\mathrm{TM}}$ Software; Sievers). Seated patients inserted a mouthpiece, inhaled orally to total lung capacity, exhaled immediately against a resistance and maintained mouth pressure at $20 \mathrm{~cm} \mathrm{H}_{2} \mathrm{O}$, displayed on a pressure gauge. The steady-state nitric oxide plateau was taken as the fractional exhaled nitric oxide (FeNO) value. By varying expiratory resistances, we measured FeNO levels at 3 expiratory flows of 50, 100 and $200 \mathrm{~mL} / \mathrm{s}$ in that order. Alveolar nitric oxide concentrations are provided as non-corrected ${ }^{\mathrm{e} 3}$ and corrected values using trumpet-shaped model and axial back diffusion ${ }^{\text {e2, e4 }}$.

After nitric oxide measurements, patients underwent prebronchodilator and postbronchodilator (ie, inhalation of $200 \mu \mathrm{g}$ of salbutamol) pulmonary function tests. Respiratory impedance was determined by impulse oscillometry system (IOS) followed by spirometric test and a nitrogen single-breath washout test. Forced vital capacity, forced expiratory volume in 1 second, and forced midexpiratory flow were determined using a ChestGraph HI-701 spirometer (Chest MI Corp., Tokyo, Japan). Spirograms were obtained in triplicate, and the best of 3 reproducible measurements was recorded, as recommended by the American Thoracic Society/European Respiratory Society ${ }^{\mathrm{e} 5}$. A nitrogen single-breath washout test was performed only before the inhalation of salbutamol to assess ventilation inhomogeneity by measuring the slope of phase 3 of the nitrogen washout curve. 
570 Respiratory impedance was determined using a Jaeger MasterScreen, IOS (Erich Jaeger,

571 Hoechberg Germany), which met standard recommendations ${ }^{\text {e6 }}$. In brief, rectangular mechanical

572 impulses containing a continuous power spectrum ranging from 0 to $100 \mathrm{~Hz}$, generated by a

573 loudspeaker at intervals of 0.2 second, were applied to the respiratory system through a

574 mouthpiece during tidal breathing. The resulting pressure and flow signals were measured next

575 to the mouthpiece and were analyzed for amplitude and phase differences using a fast Fourier

576 transform to determine respiratory resistance (Rrs) and respiratory reactance (Xrs) of the total

577 respiratory system. To reduce loss of energy in the upper airways, the chin and cheeks were

578 supported by the patients' hands. As proxies for peripheral airway function, we used the negative

579 frequency dependence of Rrs between 5 and $20 \mathrm{~Hz}$ (Rrs5-Rrs20), Xrs at $5 \mathrm{~Hz}$ (Xrs5), and

580 reactance area $(\mathrm{AX})$ that is the integral of $\mathrm{Xrs}$ from $5 \mathrm{~Hz}$ to the resonant frequency at which Xrs

581 crosses zero $^{\mathrm{e} 2, \mathrm{e} 7}$. 


\section{3 eReferences}

584 e1. Standards for the diagnosis and care of patients with chronic obstructive pulmonary 585 disease (COPD) and asthma. This official statement of the American Thoracic Society was 586 adopted by the ATS Board of Directors, November 1986. Am Rev Respir Dis. 1987;136:225-244.

587 e2. Matsumoto H, Niimi A, Jinnai M, et al. Association of alveolar nitric oxide levels with 588 pulmonary function and its reversibility in stable asthma. Respiration. 2011; 81: 311-317.

589 e3. Tsoukias NM, George SC. A two-compartment model of pulmonary nitric oxide 590 exchange dynamics. J Appl Physiol. 1998; 85: 653-666.

591 e4. Condorelli P, Shin HW, Aledia AS, Silkoff PE, George SC. A simple technique to 592 characterize proximal and peripheral nitric oxide exchange using constant flow exhalations and 593 an axial diffusion model. J Appl Physiol. 2007; 102: 417-425.

594 e5. Miller MR, Hankinson J, Brusasco V, et al. Standardisation of spirometry. Eur Respir J. $595 \quad 2005 ; 26: 319-338$.

596 e6. Oostveen E, MacLeod D, Lorino H, et al. The forced oscillation technique in clinical 597 practice: methodology, recommendations and future developments. Eur Respir J. 2003; 22: $598 \quad 1026-1041$.

599 e7. Yamaguchi M, Niimi A, Ueda T, et al. Effect of inhaled corticosteroids on small airways 600 in asthma: investigation using impulse oscillometry. Pulm Pharmacol Ther. 2009; 22: 326-332.

601 e8. Okubo K, Kurono Y, Fujieda S, et al. Japanese guideline for allergic rhinitis. Allergol Int. $602 \quad 2011 ; 60: 171-189$. 


\section{8 eFigure legends}

609 eFigure 1. Rhinitis symptom scores (RSS) in the 3 study groups. *Significant difference in the time trends

610 for RSS among the 3 treatment modalities ( $\mathrm{p}=0.004$, by 2 -way analysis of variance [ANOVA]).

$611 \dagger$ Significant changes in RSS in montelukast add-on group $(\mathrm{p}<0.001$ vs control group, by 2-way

612 ANOVA) ( $<<0.001$, by 1-way ANOVA).

613

614 

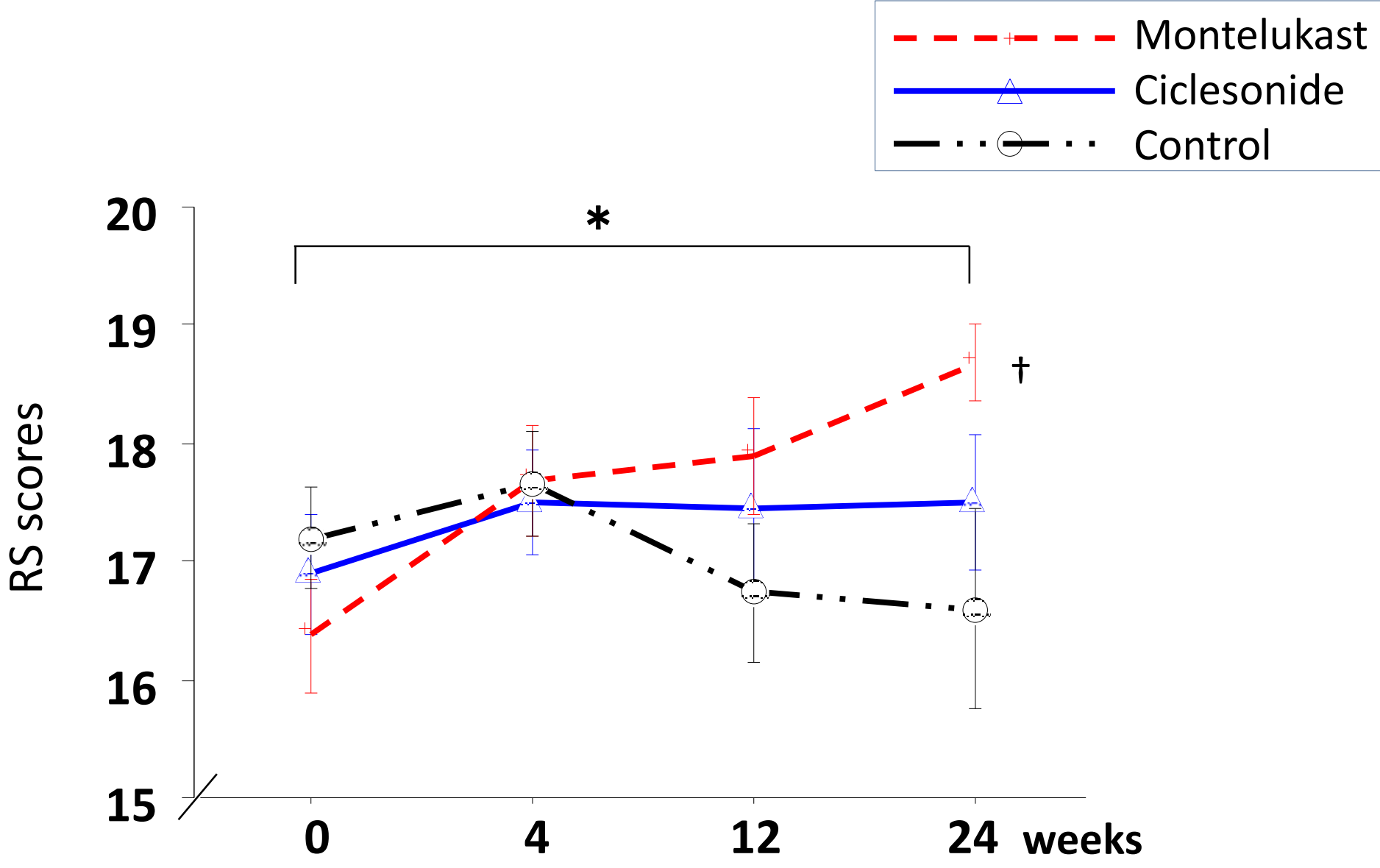

eFigure 1 
616 eTable 1.

617 Rhinitis symptom scores ${ }^{\mathrm{e} 8}$ (originally in Japanese)

618

619 A. Mean number of episodes of paroxysmal sneezing in a day

$620 \quad 1 . \geq 21$ times $\quad 2.20-11$ times $\quad 3.10-6$ times $\quad 4.5-1$ times $\quad 5$. none

621

622 B. Mean number of episodes of nasal discharge a day

$6231 . \geq 21$ times 2. 20-11 times $3.10-6$ times 4. 5-1 times 5. none

624

625 C. Nasal blockage

626 1. completely obstructed all day

627 2. severe nasal blockage causing prolonged oral breathing in a day

628 3. severe nasal blockage causing occasional oral breathing in a day

629 4. nasal blockage without oral breathing

630 5. not obstructed / no symptoms

631

632 D. Disturbance of daily activity (troubles with work, study, household work, sleep, going out, etc)

633 1. impossible

634 2. painful and complicating daily life

635 3. intermediate between 2) and 4)

636 4. few troubles

637 5. not disturbed at all 
639 eTable 2. Asthma control test (ACT) scores and distribution of control status at baseline according to the 640 treatment steps

\begin{tabular}{|l|llll|}
\hline \multicolumn{2}{|l}{} & \multicolumn{2}{c}{ Treatment steps } & \\
\cline { 3 - 4 } & & 2 and 3 & 4 and 5 & p value \\
Ciclesonide & ACT scores & $23.1 \pm 1.9$ & 16 & NS \\
$(\mathrm{n}=18)$ & total/good/no control (n) & $7 / 10 / 0$ & $0 / 0 / 1$ & $<0.01$ \\
\hline Montelukast & ACT scores & $23.3 \pm 2.4$ & $22.3 \pm 2.3$ & NS \\
$(\mathrm{n}=19)$ & total/good/no control (n) & $7 / 7 / 2$ & $1 / 2 / 0$ & NS \\
\hline Control & ACT scores & $23.7 \pm 1.7$ & $21.3 \pm 5.5$ & NS \\
$(\mathrm{n}=15)$ & total/good/no control (n) & $5 / 7 / 0$ & $1 / 1 / 1$ & NS \\
\hline
\end{tabular}

641 Data are presented as mean \pm SD.

642 Control status is defined as total when ACT score was equal to 25 points, good when ACT score was 20 643 or higher, no control when ACT score was less than 20.

644 NS; no significant difference by Wilcoxon rank-sum test or $\chi^{2}$ test.

645

646 
649 eTable 3. Summary of the results

\begin{tabular}{|c|c|c|c|c|}
\hline & & $\begin{array}{l}\text { Ciclesonide } \\
\text { add-on }\end{array}$ & $\begin{array}{l}\text { Montelukast } \\
\text { add-on }\end{array}$ & Control \\
\hline FeNO & & NS & NS & NS \\
\hline $\begin{array}{l}\text { Alveolar nitric oxide } \\
\text { concentrations }\end{array}$ & $v s$ other groups & $\begin{array}{l}\text { Significant } \\
\text { decrease } v s \\
\text { controls }\end{array}$ & NS & - \\
\hline & within the treatment modality & Decreased & Decreased & NS \\
\hline $\begin{array}{l}\text { Corrected alveolar } \\
\text { nitric oxide } \\
\text { concentrations }\end{array}$ & within the treatment modality & $\begin{array}{l}\text { Insignificantly } \\
\text { decreased }\end{array}$ & NS & NS \\
\hline $\mathrm{AX}$ & $v s$ other groups & NS & $\begin{array}{l}\text { Significant } \\
\text { decrease } v s \\
\text { controls }\end{array}$ & - \\
\hline Blood eosinophils & within the treatment modality & NS & Decreased & NS \\
\hline ACT & within the treatment modality & Improved & $\begin{array}{l}\text { Insignificantly } \\
\text { improved }\end{array}$ & NS \\
\hline
\end{tabular}

ACT: asthma control test

651 AX: reactance area at low frequencies

652 NS; no significant difference or no significant changes 